\title{
AN ALGORITHM TO BUILD CONVEX HULLS FOR 3-D OBJECTS
}

\author{
Han-Ming Chen* and Tzung-Han Lin
}

\begin{abstract}
In this paper, a new algorithm based on the Quickhull algorithm is proposed to find convex hulls for 3-D objects using neighbor trees. The neighbor tree is the data structure by which all visible facets to the selected furthest outer point can be found. The neighboring sequence of ridges on the outer boundary of all visible facets also can be found directly from the neighbor tree. This new algorithm is twice as efficient as Barber's algorithm.
\end{abstract}

Key Words: 3-D convex hull, quickhull algorithm, computational geometry.

\section{INTRODUCTION}

The convex hull of a set $\mathrm{S}$ of points is defined as the smallest convex set containing all the points in $\mathrm{S}$. It is one of the most fundamental concepts in the field of computer graphics, computational geometry, pattern recognition, computer-aided design, image processing (Robert and Faugeras, 1995), and robotics. It also plays an important role in many applications such as shape determination (Kaasalainen and Torppa, 2001), collision detection (Sherali et al., 2001), finding the minimum bounding box (O'Rourke, 1993), and constructing spherical Voronoi diagram (Sugihara, 2000).

Graham (1972) proposed an algorithm running in time complexity $O(n \log n)$ for finding the convex hull of a set of planar points. Anderson reevaluated Graham's algorithm for simplifying the computation to determine whether the second point in three consecutive points is convex or not (Anderson, 1978). Later, several quick methods based on Graham's algorithm were published to determine a 2-D convex hull for a set of planar points (Atwah et al., 1995; Atwah and Baker, 2002; Koplowitz and Jouppi, 1978). Jarvis presented a convex hull identification method that includes point deletion checks and is especially effective when the number of points on the plane is relatively small (Jarvis, 1973). In 1996, an algorithm that is a parallel adaptation of Jarvis March and the

*Corresponding author. (Tel: 886-2-23680375; Fax: 886-223631755; Email: hmchen@ntu.edu.tw)

The authors are with the Department of Mechanical Engineering, National Taiwan University, Taipei, Taiwan 106, R.O.C.
Quickhull algorithm was presented by Atwah et al. (1996).

In recent decades, at least four algorithms for finding the multi-dimensional convex hull have been proposed. They are gift wrapping, divide and conquer, incremental, and Quickhull algorithms (O'Rourke, 1993).

Chand and Kapur (1970) first presented the gift wrapping algorithm in arbitrary dimensions. In the gift wrapping algorithm, the convex hull of a point set is generated by continuing to find the hyperplane making the maximum interior angle with the adjacent facet until all facets found are closed. Assume that the number of points is $n$. The gift wrapping algorithm runs in time $O\left(n^{2}\right)$. Sugihara revised the conventional gift wrapping algorithm to increase numerical robustness and topological consistency and kept the same time complexity as the conventional algorithm (Sugihara, 1994).

The divide and conquer algorithm was first proposed by Preparata and Hong (1977) to apply a merge procedure for finding the convex hull of two non-intersecting convex hulls in two or three dimensions with $O(n \log n)$ operations. A more detailed implementation of the 3-D divide and conquer algorithm was presented by Day (1990).

O'Rouke (1993) presented a detailed implementation for the incremental algorithm. Initially, one tetrahedral hull is formed by randomly selecting four non-degeneracy points. Each time a new tetrahedron is formed with an outside point and a triangle on the old hull. Afterward this new tetrahedron is merged into the old hull and the concave parts of the merged hull are filled up. The incremental algorithm is sometimes called the beneath-beyond algorithm. Allison 
and Noga (1997) combined the divide and conquer algorithm with the incremental algorithm in computing 3-D convex hulls. In 3-D cases, the incremental algorithm requires $O(n \log n)$ expected time (Clarkson and Shor, 1988; Edelsbrunner, 1987; Kallay, 1984; Mulmuley, 1994; Preparata and Shamos, 1985; Seidel, 1990). In each iterative routine of the incremental algorithm, the merged point is randomly selected so that the vertices of the convex hull can't be found efficiently.

Generally speaking, the Quickhull algorithm is evolved from the incremental algorithm. In constructing 2-D convex hulls, Eddy (1977) presented a notion to successively partition a set of planar points into several regions with binary trees. With the "throw-away" principle, Akl and Toussaint (1978) presented an algorithm in which the furthest outside point is selected for the selected edge of the current convex hull in each iterative routine and then the selected edge is replaced with two new edges. Instead of a triangle used by Eddy and a quadrilateral used by Akl (1978) and Bykat (1978) uses a line segment to divide planar points into two regions in the first step. For general-dimension Quickhull algorithms, Barber et al., (1996) proposed detailed procedures and empirical data. His algorithm is very efficient in constructing general-dimension convex hulls.

In this paper, a new Quickhull algorithm for building 3-D convex hulls is presented. We propose some efficient routines to find a tetrahedron as the initial convex hull and then build successively the new convex hull for each new selected vertex. In finding the boundary of all visible facets for a new selected vertex, a neighbor tree is used in this paper. Because Barber's algorithm is one of the latest and efficient Quickhull algorithms, the efficiency of our algorithm is compared with that of Barber's algorithm.

\section{METHODS}

In some mathematical models, the vertex or facet enumeration is used for representing the convex hull. The vertex enumeration only contains all the vertices of the convex hull in random order. The facet enumeration lists the inside halfspace for each facet of the convex hull. In this paper, each facet and its three neighboring facets of the current convex hull are recorded in a linked list. Compared with the data structures of previous Quickhull algorithms, a much simpler data structure is proposed in this paper to raise the efficiency of our algorithm. The procedure of our algorithm is described as follows and its pseudo-code is shown in Fig. 1 starting from the main routine CONVEX_HULL().

(1) Record all the points of input point set in a linked list $L_{s}$. Remove duplicated points from $L_{s}$ with an octree. Substitute the coordinate of each point in $L_{s}$ into four formulas $x+y+z, x-y-z,-x+y-z$, and $-x-y+z$ separately. Find out a point $A$ in $L_{s}$ with the maximum value for the formula $x+y+z$ and then take out point $A$ from $L_{s}$. Repeat the same process for points $B, C$, and $D$ and remove them from $L_{s}$ one by one. Points $B, C$, and $D$ have the maximum values for formulas $x-y-z,-x+y-z$, and $-x-y+z$ respectively. Hence, points $A, B, C$, and $D$ are definitely different. The initial tetrahedron is built with points $A, B, C$, and $D$ if any three points of them are not collinear. The normal vector of each facet of the initial tetrahedron must point outward. For each facet of the initial tetrahedron, its three neighboring facets are stored in a linked list. This part of the work has been done by the function FindInitialHull() in Fig. 1.

(2) All the points in $L_{s}$ are partitioned into five sets by four facets of the initial tetrahedron in line (a) of Fig. 1. Four sets are outside the tetrahedron and the fifth set is inside the tetrahedron. For each facet $T_{n}$, if there are points in front of $T_{n}$, put $T_{n}$ in the tail of a linked list $L_{p}$. Otherwise, put $T_{n}$ in the tail of another linked list $L_{e}$. After these four facets have been put into $L_{p}$ or $L_{e}$, connect $L_{e}$ after $L_{p}$ forming a new linked list $L_{h}$. Assume that the head of $L_{h}$ is the facet $T_{j}$.

(3) The work in line (b) of Fig. 1 is described as follows. If the facet $T_{j}$ is NULL, the whole procedure has been finished and all facets in $L_{h}$ form the final convex hull. If $T_{j}$ is inside the current convex hull, remove $T_{j}$ from the linked list $L_{h}$, set the next facet in $L_{h}$ as $T_{j}$, and then go back to step (3). If there is no point in front of $T_{j}$, set the next facet in $L_{h}$ as $T_{j}$ and then go back to step (3).

(4) Among all points in front of $T_{j}$, find the point $P$ furthest from $T_{j}$. In line (c) of Fig. 1, set the facet $T_{j}$ as the root of a neighbor tree. Set three neighboring facets of $T_{j}$ as its three branches. Assume that the node $T_{k}$ is one of these three branches.

(5) For the node $T_{k}$, assume that the facet $T_{a}$ is the upper node of $T_{k}$ and $T_{a}, T_{b}$, and $T_{c}$ are three neighboring facets of $T_{k}$ in the neighbor tree. If $T_{k}$ is visible to point $P$, put $T_{b}$ and $T_{c}$ as two branches of $T_{k}$ into the neighbor tree in the same surrounding order as $T_{a}, T_{b}$, and $T_{c}$ are around $T_{k}$ on the convex hull. If $T_{k}$ has already appeared in this neighbor tree, end the work of branch $T_{k}$. If $T_{k}$ is not visible to point $P$, set $T_{k}$ as a boundary facet $B_{n}$ and end the work of branch $T_{k}$. If there is any branch $T_{k}$ whose work has not been ended, go back to step (5).

(6) Search the neighbor tree and take out the ridge shared by each boundary facet $B_{n}$ and the upper node facet of $B_{n}$ with the depth-first search. Connect each ridge with point $P$ to form a facet for 


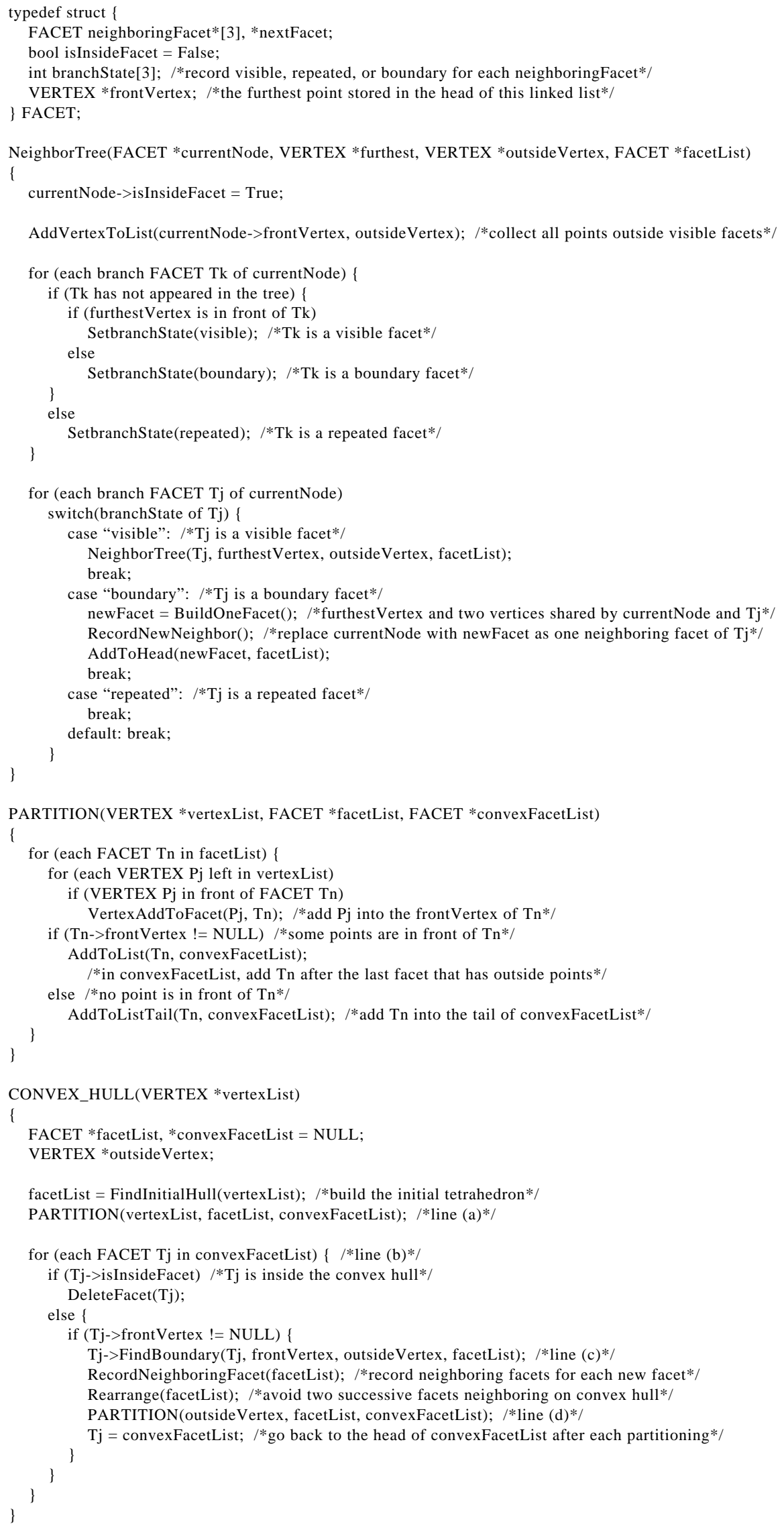

Fig. 1 The pseudo-code of our algorithm. 


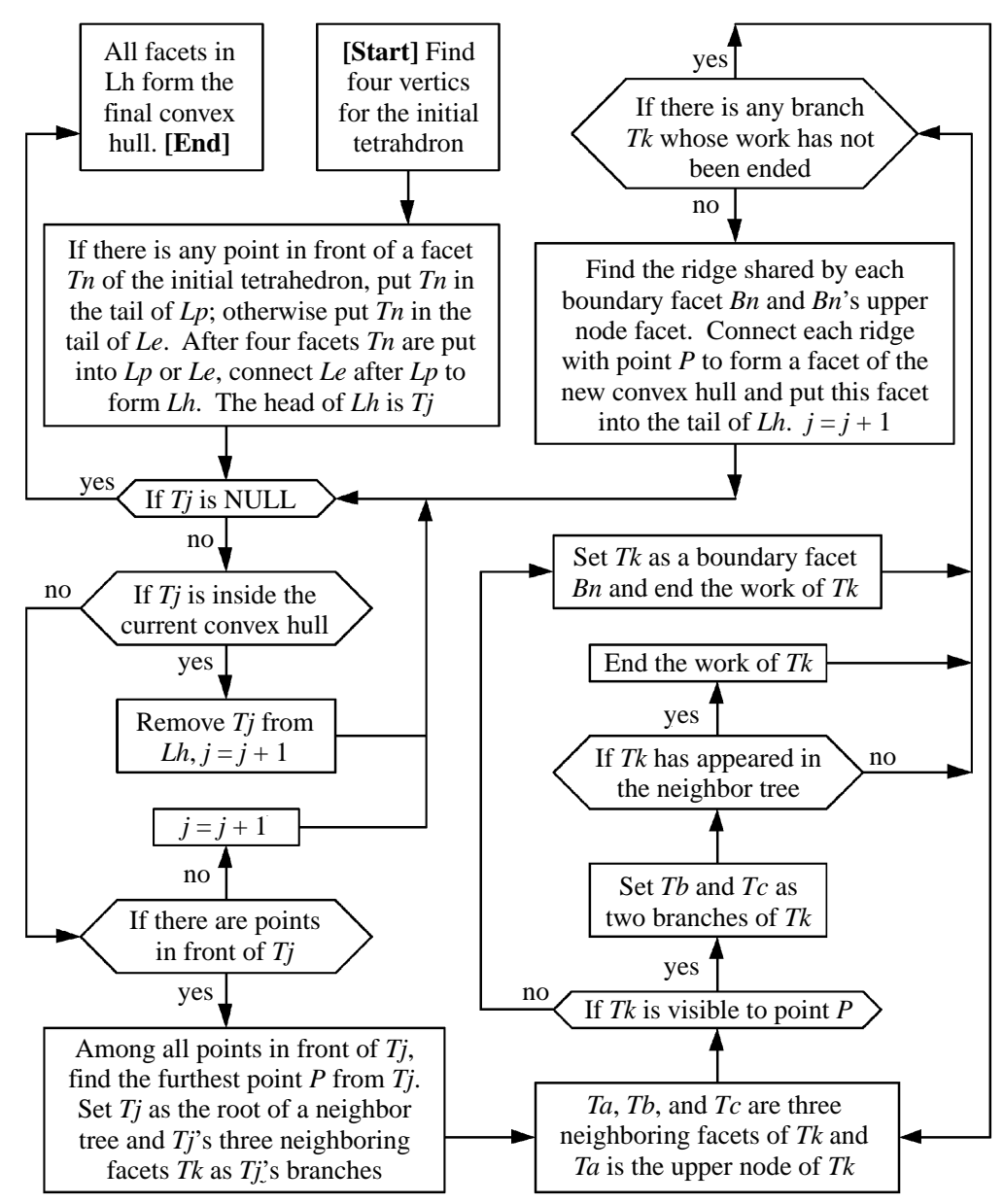

Fig. 2 The procedures of our algorithm

the new convex hull and put this facet into the tail of $L_{h}$. Collect all points outside the facets of the new convex hull.

(7) In line (d) of Fig. 1, partition all outside points with each new facet formed in step (6) using the same process as step (2). Set $j=j+1$ and go to step (3).

The procedures whole procedures of our algorithm whose starting point and terminal point are [Start] and [End] respectively is shown in Fig. 2.

For 3-D cases mentioned above, four vertices $A, B, C$, and $D$ are required to build the initial tetrahedron. In $2-\mathrm{D}$ cases, only three points $P_{1}, P_{3}$, and $P_{5}$ shown in Fig. 3 are needed to form the initial triangle. To demonstrate the whole procedure of our algorithm in a simpler way, a 2-D example is used. In order to describe one important process in our algorithm as shown in Fig. 3(b), the highest point in Fig. 3(a) is not chosen as one vertex, i.e., the point $P_{1}$, for the initial triangle.

In Fig. 3(a), all points are partitioned by facets in sequence of $T_{1}, T_{2}$, and $T_{3}$. There are points in front of $T_{2}$ and $T_{3}$ but there is no point in front of $T_{1}$.
Facet $T_{3}$ is after facet $T_{2}$ in the partitioning sequence so $T_{3}$ is put after $T_{2}$ in the linked list of Fig. 4(a) and $T_{1}$ is put in the tail of Fig. 4(a). Facet $T_{2}$ is the first element with outside points in Fig. 3(a) so $T_{2}$ is set as the root of the neighbor tree in Fig. 5(a). Among all points in front of $T_{2}$ in Fig 3(a), find the furthest point $P_{2}$ from $T_{2}$. Facets $T_{1}$ and $T_{3}$ are the neighboring facets of $T_{2}$ and not visible to $P_{2}$ so $T_{1}$ and $T_{3}$ are the boundary facets of this tree and set as the branches of the root in Fig. 5(a). The ridge shared by $T_{1}$ and $T_{2}$ is point $P_{3}$ and the ridge shared by $T_{2}$ and $T_{3}$ is point $P_{1}$. Connect $P_{2}$ to $P_{3}$ and $P_{1}$ separately to get two new facets $T_{4}$ and $T_{5}$. All points which are in front of $T_{2}$ are partitioned by $T_{4}$ and $T_{5}$ to get Fig. 3(b) and Fig. 4(b). Facet $T_{2}$ becomes an inside facet in the current convex hull and is marked with a cross in Fig. 4(b) because $T_{2}$ will not appear on the surface of the final convex hull.

After $T_{2}$ has been deleted in Fig. 3(b), $T_{3}$ becomes the first element with outside points in the linked list. Hence, set $T_{3}$ as the root of the neighbor tree and $T_{1}$ and $T_{5}$ as two branches of $T_{3}$ in Fig. 5(b). Among all points which are in front of $T_{3}, P_{4}$ is the 

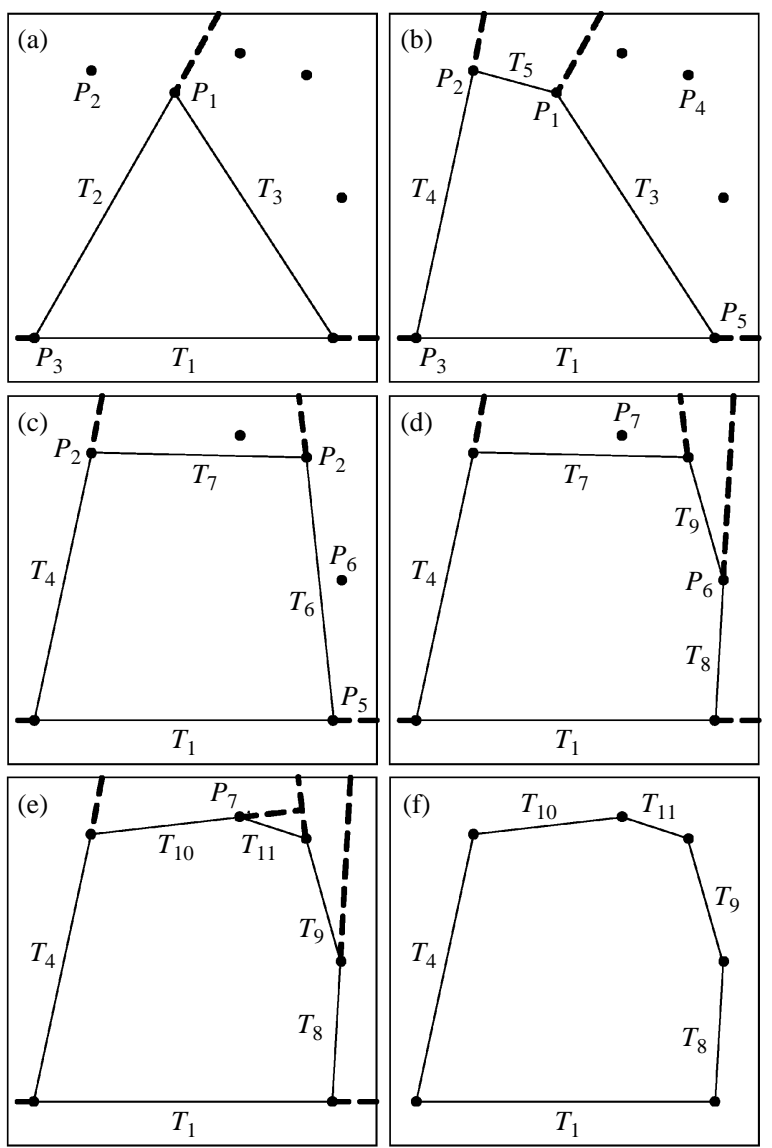

Fig. 3 A 2-D example and the processes of forming its final convex hull

(a).$T_{2} \rightarrow T_{3} \rightarrow T_{1}$

(b) $\left.\left.\mathscr{T}_{2}\right] \rightarrow T_{3}\right] \rightarrow T_{1} \rightarrow T_{4} \mid-T_{5}$

$T_{T_{3}} \rightarrow T_{1} \rightarrow T_{4} \rightarrow T_{5}$

(c) $\mathbb{Z}_{2} \rightarrow T_{6} \rightarrow T_{7}\left|T_{7} \rightarrow T_{1}\right| \rightarrow T_{4} \mid \rightarrow T_{5}$

$T_{6} \rightarrow T_{7} \rightarrow T_{1} \rightarrow T_{4} \mid \rightarrow K_{5}$

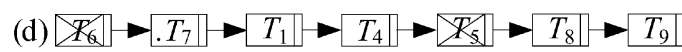

$\left.I_{7} \rightarrow T_{1} \rightarrow T_{4} \rightarrow T_{1}\right] \rightarrow T_{8} \rightarrow T_{9}$

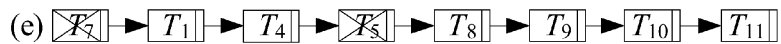

(f) $T_{1} \longrightarrow T_{4} \rightarrow T_{8} \rightarrow T_{9} \mid \rightarrow T_{10} \rightarrow T_{11}$

.$T_{n}$ : There are points in front of facet $T_{n}$

$\mathbb{F}_{k}$ : Facet $T_{n}$ is inside the current convex hull

Fig. 4 The linked lists for the 2-D example in Fig. 2

furthest point from $T_{3}$ in Fig. 3(b). Facet $T_{1}$ is not visible to $P_{4}$ so $T_{1}$ is set as a boundary facet in Fig 5 (b). Facet $T_{5}$ is visible to $P_{4}$ so $T_{5}$ is set as a visible facet. Aside from $T_{3}, T_{4}$ is another neighboring facet of $T_{5}$ and not visible to $P_{4}$ so $T_{4}$ is set as a boundary facet. Facets $T_{1}$ and $T_{4}$ are two boundary facets to $P_{4}$. The ridge between $T_{1}$ and its upper node, i.e., $T_{3}$, is $P_{5}$. Point $P_{2}$ is the ridge between $T_{4}$ and its upper

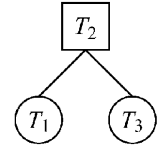

(a)

Visible facets

$\mathrm{O}$ : Boundary facets

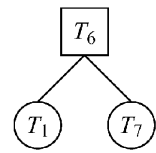

(c)

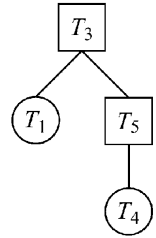

(b)

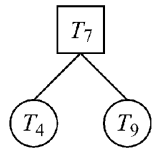

(d)
Fig. 5 Four neighbor trees for the 2-D example in Fig. 2

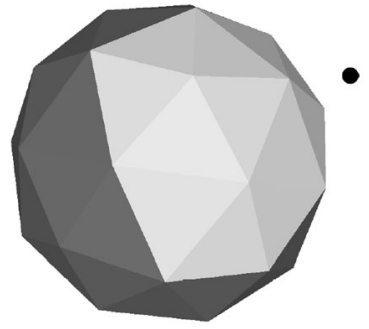

(a)

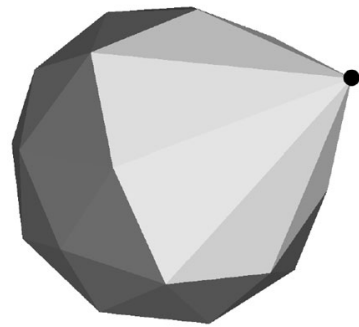

(b)
Fig. 6 (a) Light gray facets are visible to the black point and dark gray facets are not visible to the black point. (b) Merge new facets into the old convex hull to form a new convex hull

node, i.e., $T_{5}$. Connect $P_{4}$ to $P_{5}$ and $P_{2}$ to form $T_{6}$ and $T_{7}$ respectively. All points outside visible facets $T_{3}$ and $T_{5}$ are partitioned by $T_{6}$ and $T_{7}$. Facets $T_{3}$ and $T_{5}$ become inside facets in the current convex hull so they are marked with crosses in Fig 4(c). There are points outside $T_{6}$ and $T_{7}$ so $T_{6}$ and $T_{7}$ are inserted successively into the linked list after $T_{3}$ in Fig. 4(c).

Points $P_{6}$ and $P_{7}$ are the furthest points from $T_{6}$ and $T_{7}$ respectively in Fig. 3(c) and 3(d). After all inside facets in Fig. 3(e) have been deleted, all the facets of the final convex hull are composed of $T_{1}$, $T_{4}, T_{8}, T_{9}, T_{10}$, and $T_{11}$ as shown in Fig. 3(f) and 4(f). Only the vertices of the initial triangle or the furthest points can be the vertices of the final convex hull in 2-D examples.

In Fig. 6(a) that is a 3-D example, light gray facets are visible and dark gray facets are not visible to the black point respectively. All visible facets and boundary facets are found with a neighbor tree. All ridges are located on the boundary between visible facets and invisible facets. Connect the black point to the two end points of each ridge to form a new facet. All visible facets will be inside the new convex hull so they will be deleted.

In the 3-D example shown in Fig. 7(a), assume 


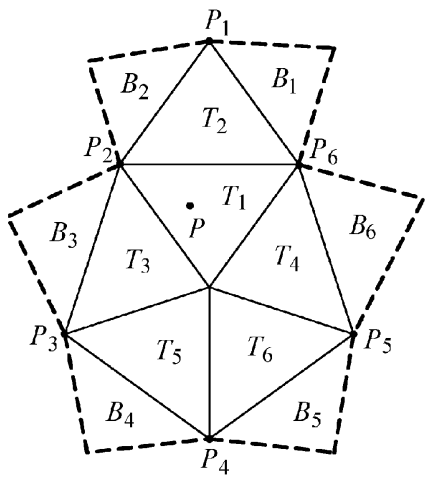

(a)

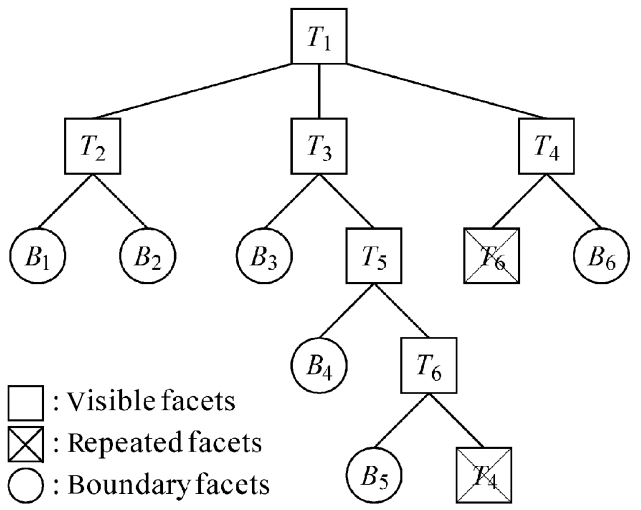

(b)

Fig. 7 (a) A 3-D example. (b) The neighbor tree of the example in (a)

that facet $T_{1}$ is set as the root of the neighbor tree in Fig. 7(b). Among all points that are in front of $T_{1}$, assume that $P$ is the furthest point from $T_{1}$. On the convex hull, facets $T_{1}$ through $T_{6}$ are visible to $P$. Facets $T_{2}, T_{3}$, and $T_{4}$ are three neighboring facets of $T_{1}$ so set these three facets as the branches of $T_{1}$.

Facets $T_{1}, B_{1}$, and $B_{2}$ are three neighboring facets of $T_{2}$ and their surrounding order around $T_{2}$ in the neighbor tree of Fig. 7(b) must be accorded with that around $T_{2}$ on the convex hull of Fig. 7(a). Facet $T_{1}$ is the upper node of $T_{2}$ so set $B_{1}$, and $B_{2}$ as two branches of $T_{2}$. Facets $B_{1}$ and $B_{2}$ are not visible to $P$ so they are boundary facets. Facets $T_{1}, B_{3}$, and $T_{5}$ are three neighboring facets of $T_{3}$ and $T_{1}$ is the upper node of $T_{3}$ so set $B_{3}$ and $T_{5}$ as two branches of $T_{3}$. In the similar way, $B_{4}$ and $T_{6}$ are set as two branches of $T_{5}$ and $B_{5}$ and $T_{4}$ are set as two branches of $T_{6}$. Facet $T_{4}$ has already appeared in the neighbor tree so $T_{4}$ is a repeated facet. Facets $B_{3}, B_{4}$, and $B_{5}$ are not visible to $P$ so they are boundary facets. Other parts of the neighbor tree are built in the same way. The boundary of all visible facets is found when every leaf node in a neighbor tree is either a boundary facet or a repeated facet.

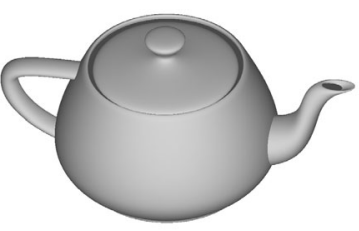

(a)

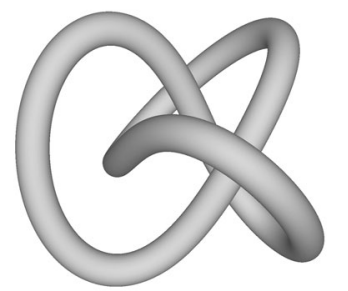

(c)

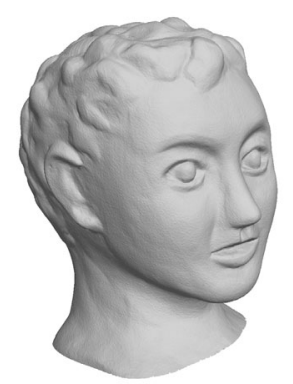

(e)

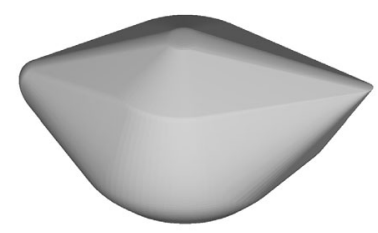

(b)

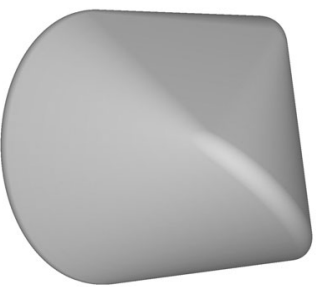

(d)

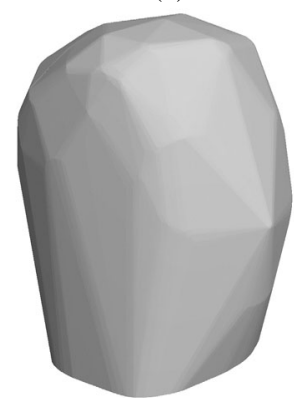

(f)
Fig. 8 Figures (a), (c), and (e) are three 3-D models. Figures (b), (d), and (f) are the convex hulls of models (a), (c), and (e) respectively

\section{IMPLEMENTATION AND COMPARISON}

Assume that the plane equation of the facet $A$ is $f_{A}(x, y, z)=0$ and the coordinate of the point $P$ is $\left(x_{P}\right.$, $\left.y_{P}, z_{P}\right)$. When building a neighbor tree in our algorithm, if $f_{A}\left(x_{P}, y_{P}, z_{P}\right)>-\varepsilon$, facet $A$ is defined as a visible facet to point $P$. When doing the partitioning process in our algorithm, if $f_{A}\left(x_{P}, y_{P}, z_{P}\right)>\varepsilon, P$ is defined as a point in front of facet $A$. In this paper, the value of $\varepsilon$ is set to 16 times of the machine epsilon (2.22E-16). Under these two conditions, degenerate cases will not cause any vague situation in our algorithm.

Models in Figs. 8(a) and 8(c) were created by the software. The model in Fig. 8(e) was made with clay and then scanned with the laser scanning system to get image data. Figs. 8(b), 8(d), and 8(f) are the convex hulls of models Figs. 8(a), 8(c), and 8(e) respectively. The last six cases in Table 1 were created by random distribution of points in a unit cube or on the surface of a unit sphere respectively.

Our algorithm has been successfully implemented in $C$ language with an Intel Pentium-4 $1.6 \mathrm{G}$ personal computer. The source code of Barber's algorithm is available via network address http://www.qhull.org/ download. In order to compare the net efficiency between Barber's algorithm and our algorithm, the time 
Table 1 The efficiency comparison between Barber's algorithm and our algorithm on nine models

\begin{tabular}{|c|c|c|c|c|c|c|}
\hline Models & $\begin{array}{l}\text { Number of } \\
\text { points }\end{array}$ & $\begin{array}{l}\text { Number of } \\
\text { point on } \\
\text { the convex } \\
\text { hull }\end{array}$ & $\begin{array}{l}\text { Number of } \\
\text { faces on the } \\
\text { convex hull }\end{array}$ & $\begin{array}{l}\text { Barber's } \\
\text { algorithm } \\
\quad(\mathrm{ms})\end{array}$ & $\begin{array}{l}\text { Our } \\
\text { algorithm } \\
(\mathrm{ms})\end{array}$ & $\begin{array}{l}\text { Efficiency } \\
\text { ratio }\end{array}$ \\
\hline Fig. 8(a) & 131,329 & 31,871 & 63,738 & 1,425 & 498 & 2.86 \\
\hline Fig. 8(c) & 288,000 & 25,867 & 51,730 & 2,140 & 793 & 2.70 \\
\hline Fig. 8(e) & 634,712 & 3,497 & 6,990 & 1,320 & 541 & 2.44 \\
\hline \multirow{3}{*}{$\begin{array}{l}\text { Points in a } \\
\text { cube }\end{array}$} & 30,000 & 171 & 338 & 54 & 24 & 2.25 \\
\hline & 300,000 & 240 & 476 & 550 & 216 & 2.55 \\
\hline & $3,000,000$ & 372 & 740 & 6,265 & 3,120 & 2.01 \\
\hline \multirow{3}{*}{$\begin{array}{c}\text { Points on a } \\
\text { sphere }\end{array}$} & 10,000 & 10,000 & 19,996 & 272 & 114 & 2.39 \\
\hline & 100,000 & 100,000 & 199,996 & 2,971 & 1,306 & 2.27 \\
\hline & $1,000,000$ & $1,000,000$ & $1,999,996$ & 45,530 & 18,215 & 2.50 \\
\hline
\end{tabular}

for reading data files is subtracted from the whole running time. Each datum in Table 1 is the average time for running the corresponding algorithm 30 times on the corresponding model.

For storing facets, double linked lists are used in Barber's algorithm, but single linked lists are used in our algorithm. As a visible facet is deleted from the double linked list in Barber's algorithm, the previous element and the next element of the deleted facet will be connected. Hence, any element in the double linked list must have two pointers to the previous element and the next element separately. In our algorithm, visible facets are found and marked in traversing the neighbor tree, and deleted in the next traverse of the linked list. Hence, the time for double linked lists is longer than that for linked lists in doing the similar work. This is the first point where our algorithm is better than Barber's algorithm.

In Barber's algorithm, the memory cell for each ridge is allocated first and then two neighboring facets and two endpoints are stored into this cell. Ridges found by Barber on the outer boundary of all visible facets are randomly stored and then these ridges form new facets with the furthest point. All new facets must be searched with a hash table to find neighboring relations among them. This extra work is not necessary in our algorithm because the neighboring sequence of ridges appears in the neighbor tree. This is the second point where our algorithm is better than Barber's algorithm.

All outer points to visible facets must be partitioned by new facets which are formed with the outer boundary of these visible facets. In Barber's algorithm, any two successively selected partitioning facets may be neighboring on the new convex hull. In our algorithm, two successively selected partitioning facets are arranged to avoid being neighboring facets on the new convex hull. Therefore, the total number of operations of substituting an outer point into the plane equation of a partitioning facet in our algorithm is less than that in Barber's algorithm. This is the third point where our algorithm is better than Barber's algorithm.

The time complexity of our algorithm is $O(n \log r)$, where $n$ is the number of input points and $r$ is the number of processed points. Although the time complexity order of our algorithm is the same as that of Barber's algorithm, our algorithm is more efficient than Barber's algorithm due to above three points in qualitative comparisons. In quantitative comparisons, the efficiency of our algorithm is over twice that of Barber's algorithm for those nine models in Table 1.

\section{CONCLUSIONS}

In this paper, the neighbor tree is proposed to find visible facets and ridges on the outer boundary of these visible facets. The data structure of our algorithm is simpler than that of Barber's algorithm. New facets found in our algorithm appear in neighboring sequence but these new facets must be arranged again in an extra process in Barber's algorithm. The order of partitioning facets to partition outer points in our algorithm is more efficient than that in Barber's algorithm. In both qualitative and quantitative comparisons, our algorithm is more efficient than Barber's algorithm.

\section{ACKNOWLEDGMENTS}

We gratefully acknowledge the support of the National Science Council of the Republic of China for this research under the grant NSC 93-2212-E-002061, and specially thank Ms. Yue-Jun Chen for making the clay model of Fig. 8(e) and Mr. Ming-Hui Lin for getting the image data of this model with the laser scanning system. 


\section{REFERENCES}

Akl, S. G., and Toussaint, G. T., 1978, "A Fast Convex Hull Algorithm," Information Processing Letters, Vol. 7, No. 5, pp. 219-222.

Allison, D. C. S., and Noga, M. T., 1997, "Computing the Three-Dimensional Convex Hull," Computer Physics Communications, Vol. 103, No. 1, pp. 74-82.

Anderson, K. R., 1978, "A Reevaluation of an Efficient Algorithm for Determining the Convex Hull of a Finite Planar Set," Information Processing Letters, Vol. 7, No. 1, pp. 53-55.

Atwah, M. M., Baker, J. W., and Akl, S. G., 1995, "An Associative Implementation of Graham's Convex Hull Algorithm," Proceedings of the Seventh IASTED International Conference on Parallel and Distributed Computing and Systems, Washington D.C., USA, pp. 273-276.

Atwah, M. M., Baker, J. W., and Akl, S. G., 1996, "An Associative Implementation of Classical Convex Hull Algorithms," Proceedings of Eighth IASTED International Conference on Parallel and Distributed Computing and Systems, Chicago, IL, USA, pp. 435-438.

Atwah, M. M., and Baker, J. W., 2002, "An Associative Static and Dynamic Convex Hull Algorithm," Proceedings of the International Parallel and Distributed Processing Symposium, Fort Lauderdale, FL, USA, pp. 15-19.

Barber, C. B., Dobkin, D. P., and Huhdanpaa, H., 1996, "The Quickhull Algorithm for Convex Hulls," ACM Transactions on Mathematical Software, Vol. 22, No. 4, pp. 469-483.

Bykat, A., 1978, "Convex Hull of a Finite Set of Points in Two Dimensions," Information Processing Letters, Vol. 7, No. 6, pp. 296-298.

Chand, D. R., and Kapur, S. S., 1970, "An Algorithm for Convex Polytopes," Journal of the Association for Computing Machinery, Vol. 17, No. 1, pp. 78-86.

Clarkson, K. L., and Shor, P. W., 1988, "Algorithms for Diametral Pairs and Convex Hulls That Are Optimal, Randomized and Incremental," Proceedings of the Fourth Annual ACM Symposium on Computational Geometry, Urbana-Champaign, IL, USA, pp. 12-17.

Day, A. M., 1990, "The Implementation of An Algorithm to Find The Convex Hull of a Set of Three-dimensional Points," ACM Transactions on Graphics, Vol. 9, No. 1, pp. 105-132.

Eddy, W. F., 1977, “A New Convex Hull Algorithm for Planar Sets," ACM Transactions on Mathematical Software, Vol. 3, No. 4, pp. 398-403.

Edelsbrunner, H., 1987, Algorithms in Combinatorial Geometry, Springer-Verlag, Heidelberg, Germany, pp. 139-176.
Graham, R. L., 1972, “An Efficient Algorithm for Determining the Convex Hull of A Finite Planar Set," Information Processing Letters, Vol. 1, No. 4, pp. 132-133.

Jarvis, R. A., 1973, "On the Identification of The Convex Hull of a Finite Set of Points in The Plane," Information Processing Letters, Vol. 2, No. 1, pp. 1821.

Kaasalainen, M., and Torppa, J., 2001, "Optimization Methods for Asteroid Lightcurve Inversion," ICARUS International Journal of Solar System Studies, Vol. 153, No. 1, pp. 24-36.

Kallay, M., 1984, "The Complexity of Incremental Convex Hull Algorithms in $\mathrm{R}^{\mathrm{d}}$," Information Processing Letters, Vol. 19, pp. 197.

Koplowitz, J., and Jouppi, D., 1978, "A More Efficient Convex Hull Algorithm," Information Processing Letters, Vol. 7, No. 1, pp. 56-57.

Mulmuley, K., 1994, Computational Geometry: An Introduction Through Randomized Algorithms, Prentice-Hall, Englewood Cliffs, NJ, USA, pp. 96-105.

O'Rourke, J., 1993, Computational Geometry in C, Cambridge University Press, New York, USA, pp. 113-167.

Preparata, F. P., and Hong, S. J., 1977, "Convex Hull of A Finite Set of Points in Two and Three Dimensions," Communications of the ACM, Vol. 20, No. 2, pp. 87-93.

Preparata, F. P., and Shamos, M. I., 1985, Computational Geometry: An Introduction, SpringerVerlay, New York, USA, pp. 95-145.

Robert, L., and Faugeras, O. D., 1995, "Relative 3D Positioning and 3D Convex Hull Computation from A Weakly Calibrated Stereo Pair," Image and Vision Computing, Vol. 13, No. 3, pp. 189-196.

Seidel, R., 1990, "Linear Programming and Convex Hulls Made Easy," Proceedings of the Sixth Annual ACM Symposium on Computational Geometry, Berkeley, CA, USA, pp. 211-215.

Sherali, H. D., Smith, J. C., and Selim, S. Z., 2001, "Convex Hull Representations of Models for Computing Collisions Between Multiple Bodies," European Journal of Operational Research, Vol. 135, No. 3, pp. 514-526.

Sugihara, K., 1994, "Robust Gift Wrapping for The Three-dimensional Convex Hull," Journal of Computer and System Sciences, Vol. 49, No. 2, pp. 391-407.

Sugihara, K., 2000, “Three-Dimensional Convex Hull As a Fruitful Source of Diagrams," Theoretical Computer Science, Vol. 235, No. 2, pp. 325-337.

Manuscript Received: Jul. 08, 2005 Revision Received: Dec. 15, 2005 and Accepted: Feb. 07, 2006 